\title{
Neural Correlate of Spatial Presence in an Arousing and Noninteractive Virtual Reality: An EEG and Psychophysiology Study
}

\author{
THOMAS BAUMGARTNER, Ph.D., ${ }^{1}$ LILIAN VALKO, M.A. ${ }^{2}$ \\ MICHAELA ESSLEN, Ph.D., ${ }^{2}$ and LUTZ JÄNCKE, Ph.D. ${ }^{2}$
}

\begin{abstract}
Using electroencephalography (EEG), psychophysiology, and psychometric measures, this is the first study which investigated the neurophysiological underpinnings of spatial presence. Spatial presence is considered a sense of being physically situated within a spatial environment portrayed by a medium (e.g., television, virtual reality). Twelve healthy children and 11 healthy adolescents were watching different virtual roller coaster scenarios. During a control session, the roller coaster cab drove through a horizontal roundabout track. The following realistic roller coaster rides consisted of spectacular ups, downs, and loops. Low-resolution brain electromagnetic tomography (LORETA) and event-related desynchronization (ERD) were used to analyze the EEG data. As expected, we found that, compared to the control condition, experiencing a virtual roller coaster ride evoked in both groups strong SP experiences, increased electrodermal reactions, and activations in parietal brain areas known to be involved in spatial navigation. In addition, brain areas that receive homeostatic afferents from somatic and visceral sensations of the body were strongly activated. Most interesting, children (as compared to adolescents) reported higher spatial presence experiences and demonstrated a different frontal activation pattern. While adolescents showed increased activation in prefrontal areas known to be involved in the control of executive functions, children demonstrated a decreased activity in these brain regions. Interestingly, recent neuroanatomical and neurophysiological studies have shown that the frontal brain continues to develop to adult status well into adolescence. Thus, the result of our study implies that the increased spatial presence experience in children may result from the not fully developed control functions of the frontal cortex.
\end{abstract}

\section{INTRODUCTION}

$S^{\prime}$ PATIAL PRESENCE is considered as "a sense of being there" which "occurs when part or all of a person's perception fails to accurately acknowledge the role of technology that makes it appear that she or he is in a physical location and environment different from her or his actual location and environment in the physical world." 1 Typically, spatial presence occurs in virtual situations created by the perception of spatial cues of the media content, not by cues of the real physical world. According to this view, television, radio, virtual reality (VR) technology and even books should be able to produce spatial presence in different ways.

${ }^{1}$ Institute for Empirical Research in Economics and Neuroeconomics; ${ }^{2}$ Institute of Psychology, Department of Neuropsychology, University of Zurich, Switzerland. 
However, although much research has been devoted to the study of spatial presence and presence using psychometrical ${ }^{2-8}$ or psychophysiological methods, ${ }^{9-12}$ only a few studies have focused on the neurophysiological and neuroanatomical underpinnings of spatial presence. Most studies in the neuroimaging domain have used VR stimuli to study cortical activations associated with spatial navigation and spatial processing. ${ }^{13-18}$ A subset of studies were particularly interested in uncovering the neural correlates of driving using driving games presented in the fMRI environment. ${ }^{19-22}$ Some studies were only interested in exploring whether a particular VR equipment could be used in the context of the fMRI and electroencephalography (EEG) environment without interfering with technical and psychological constraints. ${ }^{23,24}$ Although these studies have substantially increased our understanding of the cortical and subcortical mechanisms of spatial navigation and spatial processing, no study has directly examined the neurophysiological underpinnings of spatial presence using neuroscientific methods.

Therefore, the present study was designed to explore the relationship between spatial presence experience and cortical activations using multi-channel EEG in combination with low-resolution brain electromagnetic tomography (LORETA), allowing modeling of the underlying cortical structures which are supposed to generate the neurophysiological activations. In addition, psychometrical measures as well as psychophysiological measurements of heart rate and skin conductance were collected during an arousing and noninteractive virtual reality world depicting different roller coaster rides. Two variables were experimentally controlled or manipulated, respectively, the age of the user (children and adolescents-subject variable) and the number of monocular spatial cues (e.g., motion parallax, independent variable). Both variables have been shown to influence the spatial presence experience in psychometrical and behavioral spatial presence studies. Whereas the age of the user is negatively related with the spatial presence experience, ${ }^{25}$ the number of monocular spatial cues are positively correlated with the spatial presence experience. ${ }^{26-28}$

As no study has explicitly examined the cortical structures involved in spatial presence, we only can generate tentative hypotheses based on neuroimaging and developmental studies. For both children and adolescents, we hypothesized that the subjective spatial presence experience is enhanced in the roller coaster conditions with many monocular spatial cues compared to the condition with only few spatial cues. Because the VR world is emotional and arousing, we also expected that this enhanced spa- tial presence experience is accompanied by increased somatic and visceral reactions of the body (heart rate and skin conductance responses) as has been shown by a few psychophysiological studies in arousing VR environments. ${ }^{9}, 10$ Moreover, body reactions are known to activate brain regions involved in mapping and/or regulating the somatic and visceral state of the body (e.g., insula, secondary somatosensory corte ${ }^{29}$ ) and thus constitute an important neural correlate for conscious emotional feelings. ${ }^{30}$ Consequently, we would expect increased activation in these brain areas in the high spatial presence condition, along with other brain areas involved in emotion processing. Finally, we hypothesized both for children and adolescents that spatial presence cannot occur without activation in brain areas known to be involved in spatial processing, $13,15,18$ including in particular the intraparietal sulcus and brain regions surrounding this area of the parietal cortex. This brain region generates an egocentric view by translation of the retinal coordinates to head-centered, or even body-centered coordinates. Obviously, such an egocentric view is essential for the experience of spatial presence.

Finally, we would also expect different subjective spatial presence experience between children and adolescents, along with different brain activation patterns. An interesting structural candidate for this hypothesis is the prefrontal cortex, which is known to be involved in the executive control system of the brain, including error detection and monitoring. ${ }^{31,32}$ Based on neuroanatomical studies, it is known that the frontal cortex is among the most recent brain regions to mature, implying that the tissue itself continues to develop toward adult status well into adolescence. ${ }^{33,34}$ Thus, based on the incomplete functioning of the prefrontal cortex, we suggest that the spatial presence experience is enhanced in young children compared to adolescents, because children are less capable of cognitively controlling and monitoring the virtual experience. Accordingly, strong spatial presence experience in a noninteractive VR environment should be accompanied by reduced activation in the prefrontal cortex, and in particular in dorsal and lateral parts of this brain area, which constitute the more cognitive part of the prefrontal cortex. ${ }^{35}$

\section{METHODS}

\section{Subjects}

Twelve healthy children (five male, seven females; aged 8.4-10.7 years, mean age 9.2, SD 0.59) 
and 11 healthy adolescents (seven males, four females; aged 13.9-17.8 years, mean age 15.8, SD 1.36) volunteered for the study. All subjects were recruited from various sources, including family members, friends, or local sport clubs. All subjects were consistently right-handed and native speakers of German. Handedness was confirmed using the Annett handedness questionnaire (AHQ) and the criterion for consistent right-handedness as proposed by Annett. ${ }^{36}$ None of the subjects reported any neurological or psychiatric disease, prior head trauma, sensory impairment, or subjective cognitive impairment. All parents of the children gave informed consent and were instructed according to the ethical guidelines of the Helsinki declaration.

\section{Experimental task}

The subjects were sitting on a comfortable chair while they were viewing different roller coaster scenarios on a 17-inch computer screen placed at a distance of $1 \mathrm{~m}$ in front of them. The roller coaster scenarios were taken from commercially available roller coaster simulation software (www. nolimitscoaster.com/). The subjects wore headphones to hear the realistic driving noises with spatial cues included in the software. The viewing position of the roller coaster cab was arranged as if the subjects were sitting within the cab in the front seat while the cab was riding through the roller coaster scenario. During the initial control session (CONTROL), the cab drove through a horizontal roundabout track without any up or down movements and thus with only a few spatial cues (duration of $120 \mathrm{sec}$ ). During the following sessions, the subjects saw five different roller coaster simulations, during which several realistic rides were undertaken with spectacular ups and downs and thus with many spatial cues. The control scenario was used in order to control for various basic perceptual processing steps similar for all roller coaster scenarios, including form, color, motion, and basic spatial processing. The "realistic" roller coaster drives were divided into three different sessions: (1) initial upward driving of the roller coaster cab (UP, expectation phase; mean duration of $40 \mathrm{sec}$ ); (2) dynamic up- and downward driving of the cab, including spectacular loops (DOWN; mean duration of $63 \mathrm{sec}$ ), and (3) final horizontal drive of the cab (END; mean duration of $28 \mathrm{sec}$ ). During these rides, continuous EEG and psychophysiological measures (skin conductance response [SCR], heart rate [HR]) were obtained. Additionally, psychometric measures of spatial presence (MEC-Spatial Presence Question- naire [SPQ]) were obtained after the control condition and after the last realistic roller coaster ride.

\section{EEG recording and analysis}

EEG signals were recorded and amplified using a BrainVision 32 channel amplifier system (www. brainvision.com). Silver-silver-chloride-electrodes $(\mathrm{Ag} / \mathrm{AgCl})$ were used in association with the "Easy Cap System" (International 10-20 system, FMS Falk Minow Services, Herrsching-Breitbrunn, Germany), on which 30 electrodes were attached according to the international 10-20 system at the locations Fp1/2, F7/8, F3/4, Fz, FT7/8, FC3/4, $\mathrm{T} 7 / 8, \mathrm{C} 3 / 4, \mathrm{Cz}, \mathrm{TP} 7 / 8, \mathrm{CP} 3 / 4, \mathrm{CPz}, \mathrm{P} 7 / 8, \mathrm{P} 3 / 4$, $\mathrm{Pz}, \mathrm{O} 1 / 2, \mathrm{Oz}$ and referenced to FCz. The electrooculogram (EOG) was recorded from two additional electrodes placed below the outer canthus of the eyes. BrainVision Recorder and Analyzer (BrainProducts, Germany) were used to record (electrode impedance $<5 \mathrm{k} \Omega, 0.5-70 \mathrm{~Hz}, 500$ samples/sec) and analyze the data. After recording the 5-min eyes-closed resting EEG, recording of EEG during the experimental sessions was performed. The total EEG recording time (including prestimulation recordings) was about $40 \mathrm{~min}$.

All recorded EEG epochs were carefully and individually checked for artifacts (e.g., eye blinks, horizontal and vertical eye movements, muscle artifacts) by visual inspection. Epochs that were associated even with small changes in the horizontal or vertical EOG channel within the reference or test interval were rejected (amplitude minimum, -100 $\mu \mathrm{V}$; maximum, $100 \mu \mathrm{V})$. The average number of artifact-free trials for all conditions was 80 . These artifact-free epochs were recomputed to the average reference. ${ }^{37,38}$ Furthermore, a discrete Fourier transformation algorithm was applied to all extracted artifact-free epochs of data that were $2.048 \mathrm{sec}$ in duration each. The power spectrum of $1.5-30 \mathrm{~Hz}$ was calculated for each single epoch and then averaged across epochs. Finally, according to the hypothesis, 12 electrodes were collapsed into three frontal and three parietal electrode clusters: frontal left (F3/FC3), frontal midline $(\mathrm{Fz} / \mathrm{FCz})$, frontal right (F4/FC4), parietal left (CP3/P3), parietal midline $(\mathrm{CPz} / \mathrm{Pz})$, and parietal right $(\mathrm{CP} 4 / \mathrm{P} 4)$.

According to the surface EEG analysis in this paper, we were mainly interested in studying the changes in the Alpha band $(8-13 \mathrm{~Hz})$ because changes in the power of the Alpha band have been shown to be valid indicators of cortical activation/deactivation within the frontal and parietal lobe (i.e., power in the Alpha band is negatively related to activity). ${ }^{39-41}$ The percentage change in 
Alpha band power between the control condition (CONTROL roundabout riding) and the three sessions of realistic roller coaster riding (UP, DOWN, and END) were calculated using the following formula: event-related desynchronization/eventrelated synchronization $(\mathrm{ERD} / \mathrm{ERS})=[$ (band power reference - band power test)/(band power reference) $] \times 100.42$ Note that positive values indicate a relative power decrease in the Alpha band (ERD) and negative values indicate a relative power increase (ERS). Thus, ERD indicates cortical activation, whereas ERS indicates cortical deactivation. These ERD/ERS at the six electrode clusters were thereafter used for further statistical analyses.

\section{Psychophysiological and psychometrical measurements}

According to Meehan, ${ }^{10}$ physiological presence means that a user is "responding physiologically to the mediated environment in a manner consistent with human response to similar real situations." Therefore, in addition to EEG, measurements of HR and SCR were made during the experiment by using a commercially available device (PAR-PORT; Hogrefe Company, Germany). For SCR recording, electrodes were attached to the thenar and hypothenar areas on the palm of the left hand. Quantification of SCRs entailed measurement and summation of the SCR amplitude and division by the length of the conditions in seconds due to different durations of the four conditions. In addition, $\log$-transformation $(\log [\mathrm{SCR}+1])$ was used to normalize the SCR amplitude data. Furthermore, the self-location subscale of the MEC-SPQ questionnaire was used to obtain psychometric measures of spatial presence (MEC-SPQ ${ }^{43}$ ). This questionnaire (eight items, five-point scales ranking from " $1=\mathrm{I}$ do not agree at all" to " $5=$ I agree fully") was administered after the control condition and immediately after the last of the five roller coaster rides. The scale measures the subjective impression how much the subjects felt to be in the middle of the action of the roller coaster ride rather than merely observing it. The eight questions were carefully explained to the adolescents, but in particular to the children to ensure that they were able to understand and answer these questions properly.

\section{LORETA inverse solution}

For the purpose of displaying intracerebral EEG data, in addition to the ERD/ERS two-dimensional scalp map analysis, the LORETA inverse solution method was employed, ${ }^{47,48}$ which computes, from the recorded scalp electric potential differences, the three-dimensional distribution of the electrically active neuronal generators in the brain as a current density value $\left(\mathrm{A} / \mathrm{m}^{2}\right)$ at each voxel. LORETA solves the inverse problem based on the assumption that the smoothest of all possible activity distributions is the most plausible one. This assumption is supported by electrophysiology, where neighboring neuronal populations show highly correlated activity. ${ }^{49}$ The version of LORETA employed here used a three-shell spherical head model registered to the digitized Talairach and Tournoux $^{50}$ atlas (Brain Imaging Centre, Montreal Neurological Institute). The registration between spherical and Talairach and Tournoux head geometry used the realistic EEG electrode coordinates reported by Towle et al. ${ }^{51}$ Further, the source solution space was limited to cortical gray matter and hippocampi according to digitized probability atlas provided by the Montreal Neurologic Institute (number of voxels, 2394; voxel dimension, $7 \mathrm{~mm}^{3}$ ). Voxels were defined as gray matter if their probability of being gray matter was (a) greater than $33 \%$, (b) exceeded the probability of being white matter, and (c) exceeded the probability of being cerebrospinal fluid. It has been shown in numerous experiments that the method is capable of correct localization of primary sensory cortices, epileptic foci, language processing, face processing, and emotion processing areas with fairly low errors. ${ }^{52,53}$

Whole brain analysis using voxelwise paired $t$-tests examined differences between the CONTROL and DOWN conditions separately for children and adolescents in six frequency bands according to $\mathrm{Ku}$ bicki et al.54: Delta $(1-3 \mathrm{~Hz})$, Theta $(4-7 \mathrm{~Hz})$, Alpha $(8-12 \mathrm{~Hz})$, Beta1 (13-18 Hz), Beta2 (19-21 Hz), and Beta $3(22-30 \mathrm{~Hz})$. Based on current knowledge about the functional significance of these EEG bands, we take the Beta bands as indicating neural activation while increasing power in the Alpha and Theta band generally indicate decreased neural activation. ${ }^{55}$ Due to the conservative non-parametric randomization statistic used by the LORETA-Key software (corrected for multiple tests) we will report and discuss condition differences if the $p$ value is $<0.10$. Note that this significance criterion is much more conservative than the significance criterion used in most neuroimaging experiments exploring higher-order cognitive or emotional functions of the human brain. In most cases, these studies report results not corrected for multiple testing.

\section{Statistical analysis}

The ERD/ERS values were analyzed with a fourway ANOVA with the following repeated measure- 
ments factors: "roller coaster ride" (UP, DOWN, END), "region" (frontal vs. parietal), and "hemisphere" (left, midline, and right). In addition, a between-subjects factor was used to compare children with adolescents. Furthermore, two three-way ANOVA's with repeated measurements on each factor ("roller coaster ride," UP, DOWN, END; "region," frontal, parietal; "hemisphere," left, midline, right) were conducted separately for children and adolescents. For each psychophysiological measurement (HR and SCR), two-way ANOVAs were performed with the factors "roller coaster ride" (CONTROL, UP, DOWN, END) as repeatedmeasurement factor and "group" (children vs. adolescents) as a grouping factor. To analyze the self-location scale of the MEC-SPQ, which was answered by the subjects after the control condition and after the five roller coaster rides, a repeatedmeasures ANOVA was conducted with the factors "roller coaster ride" (control, roller coaster ride) and "group" (group 2: children, adolescents). For all repeated measurement ANOVAs the multivariate approach for factors comprising more than two conditions was used to avoid anticonservative estimation of F-values due to possible violations of homoscedasticity. ${ }^{44}$ Because it is more important to demonstrate the empirical effect of a treatment rather than to calculate $p$ values, we also report effect size measures independent from sample size. ${ }^{45}$ An effect size measure ETA ${ }^{2}$ was calculated, which is the estimated variance due to the treatment in relation to the total variance. Thus, ETA ${ }^{2}$ describes the variance which is due to the experimental effect. All statistical analyses were performed using the statistical software package SPSS PC (version 11.5). Results were considered significant at the level of $p<0.05$. In case of a significant multivariate effect post hoc paired $t$-tests were computed using the Bonferroni correction according to Holm. ${ }^{46}$

\section{RESULTS \\ Self-location rating (MEC-SPQ)}

The highly significant main effect of "roller coaster ride" $\left(\mathrm{F}_{(1,21)}=35.34, p<0.001, \mathrm{ETA}^{2}=0.627\right)$ was due to the higher rating of self-location during the roller coaster rides compared to the control condition for both children and adolescents. Furthermore, a group effect was observed $\left(\mathrm{F}_{(1,21)}=\right.$ $\left.4.30, p=0.05, \mathrm{ETA}^{2}=0.170\right)$, indicating that the children experienced stronger Spatial Presence than the adolescents.

\section{Skin conductance responses and heart rate}

As Figure 1 illustrates, there was a highly significant main effect of "roller coaster ride" $\left(\mathrm{F}_{(3,19)}=\right.$ $\left.17.55, p<0.001, \mathrm{ETA}^{2}=0.735\right)$, indicating the largest SCR sum amplitude for UP, intermediate for DOWN and lowest for END and CONTROL. In addition, SCR amplitude and the subjective spatial presence experience as measured with the MECSPQ subscale showed one significant correlation and one trend for a significant positive correlation (SCR UP/Self-Location: $\mathrm{r}=0.409, p=0.053$; SCR DOWN/Self-Location: $r=0.485, p<0.05$ ), demonstrating that subjects who rated high on the MECSPQ subscale also showed increased SCRs in the most arousing conditions of the experiment (UP and DOWN). Surprisingly, no significant differences regarding HR measures were observed.

\section{ERD/ERS of alpha power (children and adolescents)}

The four-way ANOVA for the dependent variable ERD/ERS revealed a significant four-way interaction between all independent variables ("roller coaster ride" * "group"* "region" * "hemisphere": $\left.\mathrm{F}_{(4,18)}=4.2, p=0.014, \mathrm{ETA}^{2}=0.485\right)$; thus, all other main effects or interactions should be interpreted with reluctance. However, "region" and "region" * "roller coaster ride" showed a strong effect on the ERD/ERS values ("region": $\mathrm{F}_{(1,21)}=14.4$, $p<0.001, \mathrm{ETA}^{2}=0.407$ and "roller coaster ride" * "region": $\left.\mathrm{F}_{(2,20)}=11.5, p<0.001, \mathrm{ETA}^{2}=0.536\right)$, indicating increased ERD (cortical activity) at parietal electrode clusters compared to the frontal electrode

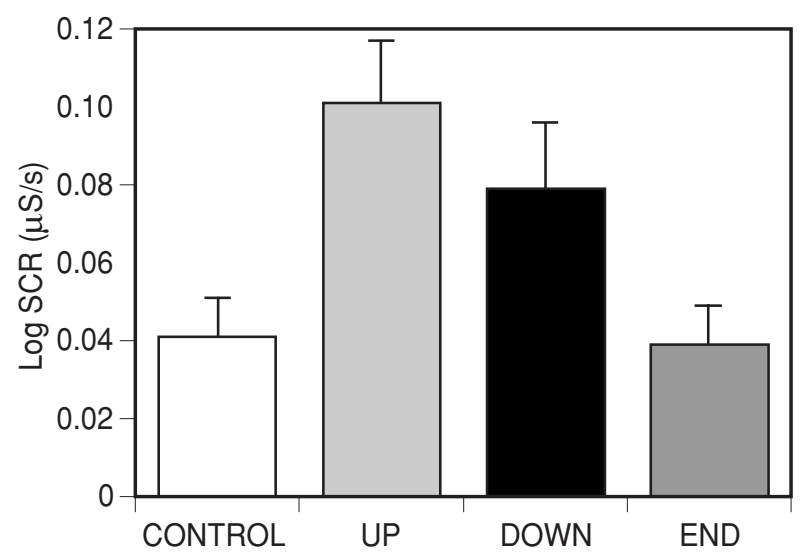

FIG. 1. Depicted are means $( \pm S E)$ of the $\log [S C R+1]$ in $\mu \mathrm{S}$ per second. Findings demonstrate the largest skin conductance response (SCR) for UP, intermediate for DOWN, and significantly reduced SCR for CONTROL and END. No group differences between children and adolescents were observed. 
clusters and this effect was most pronounced in the DOWN (dynamic roller coaster ride) condition. In addition, the main effect of "hemisphere" $\left(\mathrm{F}_{(2,20)}=\right.$ $\left.4.7, p=0.021, \mathrm{ETA}^{2}=0.320\right)$ and the interaction effect of "region" * "hemisphere" $\left(\mathrm{F}_{(2,20)}=5.8, p=\right.$ $\left.0.01, \mathrm{ETA}^{2}=0.366\right)$ demonstrated topographically the largest positive percentage change (ERD) at the midline parietal electrode cluster. Most interestingly, a significant interaction between "region" * "group" was observed $\left(\mathrm{F}_{(1,21)}=4.3, p=0.05, \mathrm{ETA}^{2}=\right.$ 0.171 ), which was qualified by increased frontal ERD for adolescents compared to children. In other words, children showed reduced activity at frontal electrodes (Fig. 2). Since the four-way interaction was significant and four-way interactions are difficult to interpret, two additional three-way ANOVA's were calculated separately for children and adolescents.

\section{ERD/ERS of alpha power (children)}

The three-way ANOVA revealed two main effects for "roller coaster ride" $\left(\mathrm{F}_{(2,10)}=4.1, p=0.05\right.$, ETA $\left.^{2}=0.451\right)$ and "region" $\left(\mathrm{F}_{(1,11)}=11.9, p<0.01\right.$, $\left.\mathrm{ETA}^{2}=0.519\right)$ and a significant interaction effect for "region" * "roller coaster ride" $\left(\mathrm{F}_{(2,10)}=7.8, p<0.01\right.$, $\mathrm{ETA}^{2}=0.611$ ). Figure 3 depicts this significant interaction which clearly demonstrates the dissociation between frontal and parietal electrodes during the roller coaster ride in children. They showed the strongest activations at parietal leads during the DOWN condition and significantly reduced cortical activity both in the UP and END conditions. By contrast, frontal electrodes demonstrated a different pattern which was qualified by an increase in

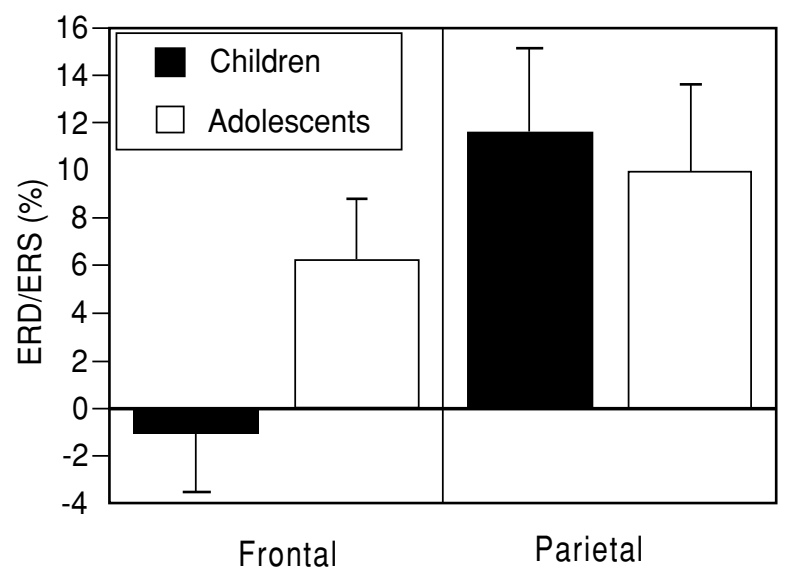

FIG. 2. Illustrated are means $( \pm \mathrm{SE})$ of the significant interaction effect of REGION*GROUP ( $p=0.05)$, which is qualified by reduced activity at frontal electrodes in children compared to adolescents.

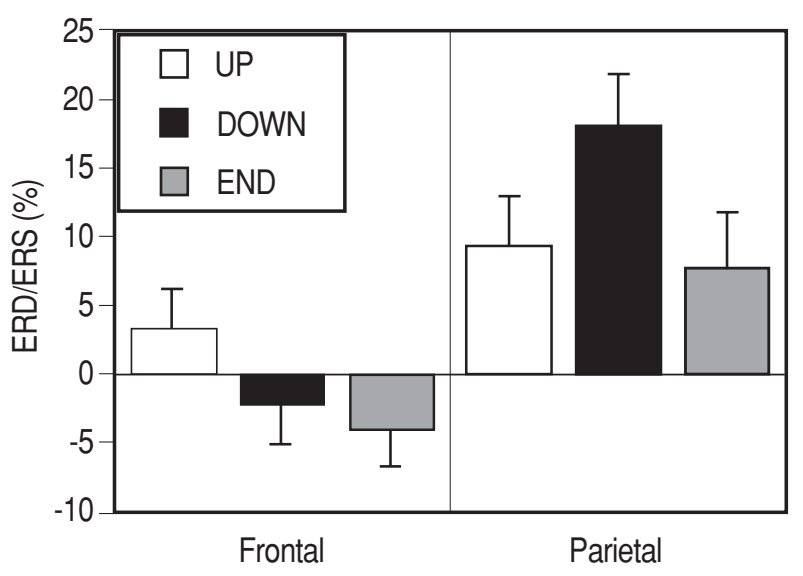

FIG. 3. Depicted are means $( \pm \mathrm{SE})$ of the highly significant interaction effect of REGION*COND $(p<0.01)$ in children, indicating a clear dissociation between frontal and parietal electrode clusters, which is most pronounced in the DOWN (dynamic roller coaster ride) condition.

cortical activation in the UP condition and a significant cortical decrease of activation in the DOWN and END conditions. In addition, both in the DOWN and END conditions, the frontal activation patterns compared to the parietal patterns differed significantly.

\section{ERD/ERS of alpha power (adolescents)}

The three-way ANOVA showed a significant interaction effect of "region" * "roller coaster ride" $\left(\mathrm{F}_{(2,9)}=4.5, p=0.043, \mathrm{ETA}^{2}=0.503 ;\right.$ Fig. 4), indicating,

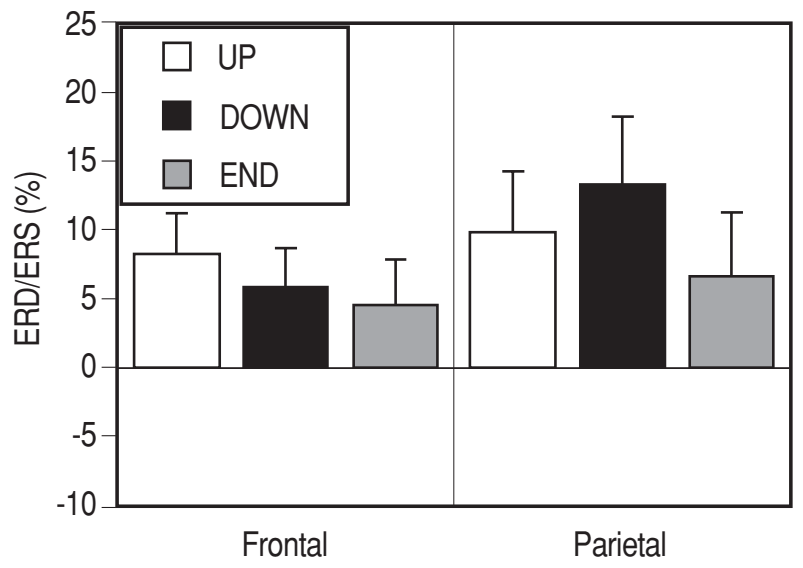

FIG. 4. Depicted is the significant interaction effect of REGION*COND $(p=0.043)$ in adolescents (means $\pm \mathrm{SE})$, demonstrating the highest event-related desynchronization (ERD) in the DOWN and UP condition. In contrast to children, no event-related synchronization (ERS; cortical deactivation) could be observed at frontal electrode clusters. 
similar to children, the highest ERD in the DOWN and UP condition and significantly reduced ERD in the END condition at parietal electrode clusters. In contrast to the activation pattern of the children, adolescents showed increased cortical activation at frontal electrodes in all three conditions (UP, DOWN, END). Additionally, frontal and parietal ERD's differentiated in the DOWN condition. Table 1 summarizes the means $( \pm \mathrm{SE})$, whereas Figure $5 a, b$ illustrates the scalp maps of the ERD and ERS values for both groups broken down for all conditions. Finally, ERD/ERS difference maps were calculated (Fig. 5c) between children and adolescents, illustrating the different activation patterns of children and adolescents in the three sessions of the roller coaster ride.

\section{LORETA}

As expected, the statistical comparison between the CONTROL and DOWN condition (separately for children and adolescents) using voxel-wise paired $t$-tests for all frequency bands revealed significant differences in the Alpha-band $(8-12 \mathrm{~Hz})$ for both groups. Furthermore, two bands, the Theta band $(4-7 \mathrm{~Hz})$ in children and the Beta2 band $(19-21 \mathrm{~Hz})$ in adolescents, showed trends towards significance on the $10 \%$ level. No other significant results or trends towards significance in the other frequency bands were observed (all $p>0.25$ ). Table 2 summarizes all findings separately for children and adolescents. Alpha band activity was decreased both in children and adolescents in the DOWN condition mainly in parietal and occipital brain regions, indicating increased activity in the DOWN condition in regions known to be essen- tially involved in processing of visual spatial information. In children, this activity increase was most prominent on the right hemisphere while in adolescents a more bilateral activation pattern was found (Figs. 6 and 7, first row). A further decrease in the Alpha band activity in both groups in the DOWN condition was observed in the posterior cingulate gyrus. Interestingly, children but not adolescents demonstrated increased Alpha band activity (indicating decreased neural activity) in the DOWN condition in frontal regions (right inferior frontal gyrus and right middle frontal gyrus) known to be involved in various executive functions. In addition, in children the Alpha band activity was increased during the DOWN condition bilaterally in the insula and the left postcentral gyrus (Fig. 6, first row). Noteworthy, no Alpha band increase in the DOWN condition could be observed in adolescents. In contrast, adolescents revealed a marked increase of Beta2 activity in left precentral gyrus, left inferior frontal gyrus and left insula during the DOWN condition (Fig. 7 second row). Finally, in the DOWN condition children showed decreased Theta activity in right inferior parietal lobe and right precuneus and increased Theta activity in bilateral superior frontal gyrus and left middle temporal gyrus, respectively (Fig. 6, second row).

\section{DISCUSSION}

The major aim of the present study was to explore whether different spatial presence experiences are associated with typical cortical activation patterns. For this, different VR roller coaster scenarios were used in the context of EEG measure-

\begin{tabular}{|c|c|c|c|c|c|}
\hline \multirow[b]{2}{*}{ Condition } & \multirow[b]{2}{*}{ Region } & \multicolumn{2}{|c|}{ Children } & \multicolumn{2}{|c|}{ Adolescents } \\
\hline & & Mean & $S E$ & Mean & $S E$ \\
\hline \multirow[t]{2}{*}{ UP } & Frontal & 3.21 & 2.92 & 8.24 & 2.95 \\
\hline & Parietal & 9.21 & 3.51 & 9.91 & 4.37 \\
\hline \multirow[t]{2}{*}{ DOWN } & Frontal & -2.25 & 2.94 & 5.91 & 2.76 \\
\hline & Parietal & 17.95 & 3.81 & 13.31 & 4.91 \\
\hline \multirow[t]{2}{*}{ END } & Frontal & -4.04 & 2.74 & 4.54 & 3.32 \\
\hline & Parietal & 7.67 & 3.95 & 6.59 & 4.70 \\
\hline
\end{tabular}

ERD, event-related power desynchronization; ERS, event-related power synchronization; SE, standard error. 
Table 2. LORETA Results of Significant Differences in Brain Electrical Activity during the DOWN COMPARED to THE CONTROL CONDITION SEPARATELY FOR CHILDREN AND ADOLESCENTS

\begin{tabular}{|c|c|c|c|c|c|c|}
\hline \multicolumn{5}{|c|}{ Frequency band } & \multicolumn{2}{|c|}{ Brain region (Brodmann area) } \\
\hline Group & Name & $\begin{array}{l}\text { Range } \\
(\mathrm{Hz})\end{array}$ & & & Left & Right \\
\hline \multirow[t]{10}{*}{ Children } & Theta $^{\mathrm{d}}$ & $4-7$ & & $\downarrow$ a & & $\begin{array}{l}\text { Inferior parietal lobe }(7) \\
\quad(39,-67,43)^{\mathrm{b}}\end{array}$ \\
\hline & & & & $\downarrow$ & & $\begin{array}{l}\text { Superior parietal gyrus / } \\
\text { precuneus }(7) \\
\quad(4,-60,43)\end{array}$ \\
\hline & & & $\uparrow$ & & $\begin{array}{l}\text { Superior frontal gyrus (6) } \\
\quad(-10,-4,71)\end{array}$ & $\begin{array}{l}\text { Superior frontal gyrus (6) } \\
\quad(11,-4,71)\end{array}$ \\
\hline & & & $\uparrow$ & & $\begin{array}{l}\text { Temporal gyrus }(21 / 20 / 38) \\
\quad(-59,3,-6)\end{array}$ & \\
\hline & Alphac & $8-12$ & & $\downarrow$ & & $\begin{array}{l}\text { Precuneus }(19,7) \\
\quad(25,-88,36)\end{array}$ \\
\hline & & & & $\downarrow$ & & $\begin{array}{l}\text { Posterior cingulate }(29 / 30) \\
\quad(4,-39,22)\end{array}$ \\
\hline & & & $\uparrow$ & & & $\begin{array}{l}\text { Middle frontal gyrus }(6 / 8 / 9) \\
\quad(32,10,57)\end{array}$ \\
\hline & & & $\uparrow$ & & & $\begin{array}{l}\text { Sub-gyral frontal lobe (6) } \\
\quad(25,-4,57)\end{array}$ \\
\hline & & & $\uparrow$ & & $\begin{array}{l}\text { Insula }(13) \\
\qquad(-38,-18,15)\end{array}$ & $\begin{array}{l}\text { Insula }(13) \\
\qquad(39,-4,8)\end{array}$ \\
\hline & & & $\uparrow$ & & $\begin{array}{l}\text { Postcentral gyrus }(3 / 43) \\
\quad(-66,-11,22)\end{array}$ & $\begin{array}{l}\text { Inferior frontal gyrus }(9 / 44 / 45) \\
\quad(60,10,29)\end{array}$ \\
\hline \multirow[t]{6}{*}{ Adolescents } & Alphac & $8-12$ & & $\downarrow$ & $\begin{array}{l}\text { Posterior cingulate (29) } \\
\quad(-3,-39,22)\end{array}$ & $\begin{array}{l}\text { Posterior cingulate (29) } \\
\quad(4,-39,22)\end{array}$ \\
\hline & & & & $\downarrow$ & $\begin{array}{l}\text { Cingulate gyrus (31) } \\
\quad(-3,-46,29)\end{array}$ & $\begin{array}{l}\text { Cingulate gyrus (31) } \\
\quad(4,-46,29)\end{array}$ \\
\hline & & & & $\downarrow$ & $\begin{array}{l}\text { Superior parietal gyrus / } \\
\text { precuneus }(7) \\
(-10,-67,57)\end{array}$ & $\begin{array}{l}\text { Superior parietal gyrus / } \\
\text { precuneus }(7) \\
(11,-63,57)\end{array}$ \\
\hline & Beta2d & $19-21$ & $\uparrow$ & & $\begin{array}{l}\text { Precentral gyrus }(4) \\
\quad(-59,-4,22)\end{array}$ & \\
\hline & & & $\uparrow$ & & $\begin{array}{l}\text { Inferior frontal gyrus }(44 / 45) \\
\quad(-52,10,22)\end{array}$ & \\
\hline & & & $\uparrow$ & & $\begin{array}{l}\text { Insula }(13) \\
\quad(-38,-11,1)\end{array}$ & \\
\hline
\end{tabular}

aDownward arrows indicate decreases and upward arrows indicate increases of electrical activity for DOWN compared to CONTROL regarding a specific frequency band.

bCoordinates are defined in the standard stereotaxic space of Talairach and correspond to the observed activation maxima. Brodmann areas describe the extent of the activated brain region.

$c p<0.05$ (voxel statistic, corrected for multiple comparison).

$\mathrm{d} p<0.10$ (voxel statistic, corrected for multiple comparison).

LORETA, low-resolution brain electromagnetic tomography.

ments. One scenario was a simple horizontal roundabout tour (low spatial presence condition), whereas the other scenarios were more realistic roller coaster rides that included the typical ups, downs, and loops (high spatial presence condition). For the more realistic roller coaster scenario, we also analyzed different sessions of the ride (UP, DOWN, and END), because we hypothesized that 


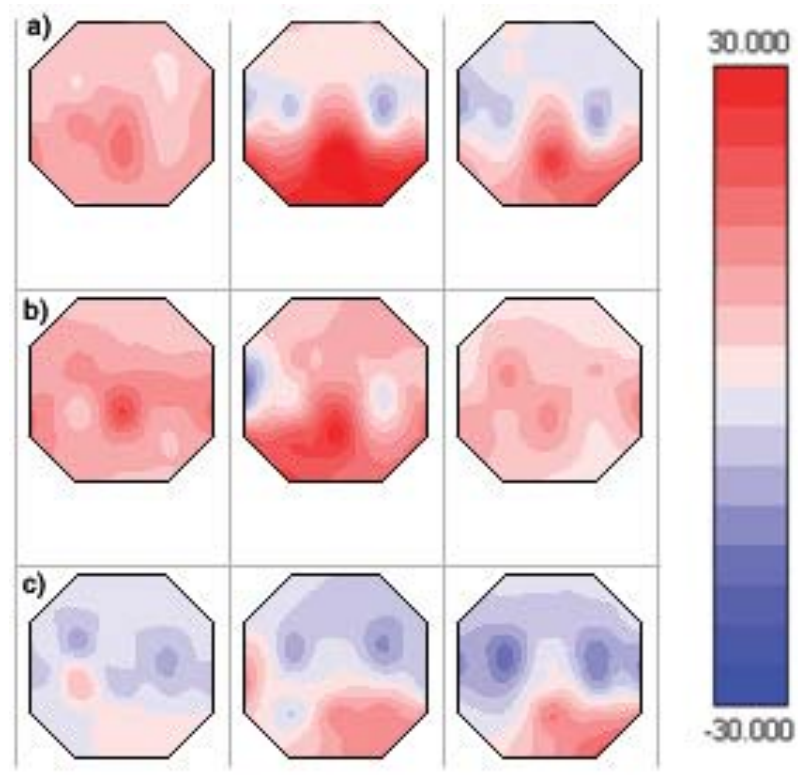

FIG. 5. Event-related desynchronization/ event-related synchronization maps of children (a), adolescents (b), and children minus adolescents (c). $(a, b)$ Depicted is the percentage change in alpha band power between the control condition (CONTROL) and the three sessions of the realistic roller coaster riding (UP, DOWN, and END; from the left to the right column); note that red colors stand for ERD (cortical activation) and blue colors for ERS (cortical deactivation). (c) ERD/ERS difference maps between children and adolescents in the UP, DOWN, and END condition (from left to right). Maps illustrate that children compared to adolescents reacted to the roller coaster paradigm with increased cortical activation at parietal occipital electrodes on the one side and decreased cortical activation at frontal electrodes on the other side.

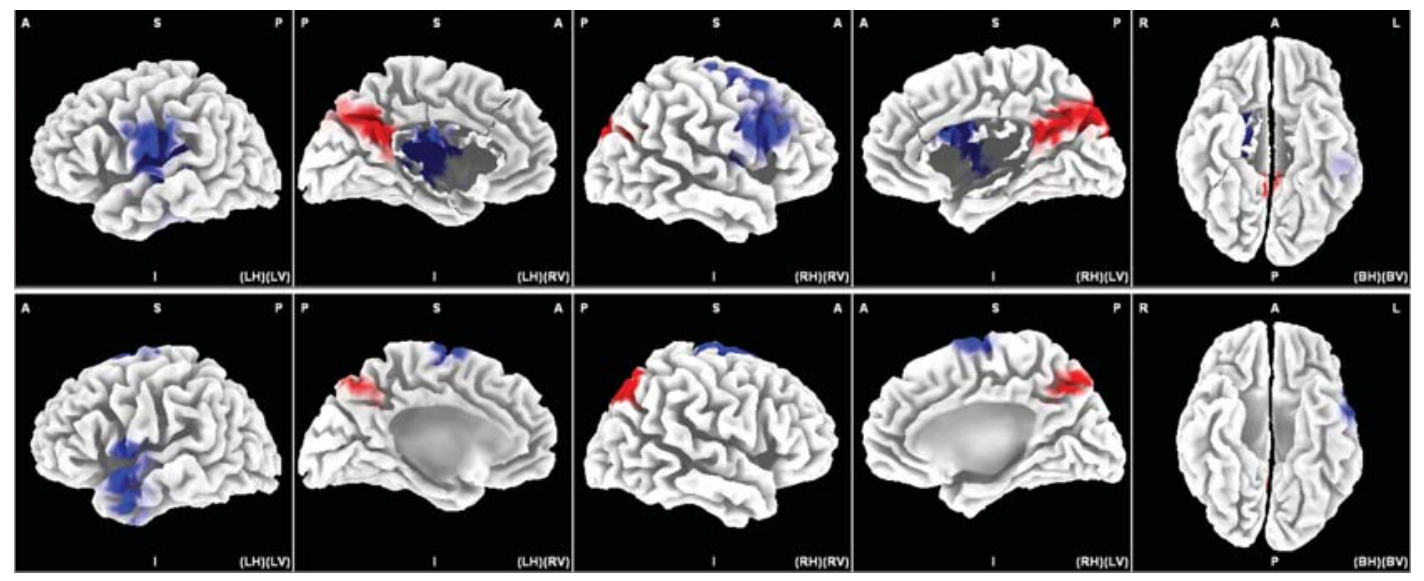

FIG. 6. Low-resolution brain electromagnetic tomography (LORETA) functional images of regional electric activity differences for the comparison "DOWN minus CONTROL" in children. (First row) Alpha (8-12 Hz) band. (Second row) Theta $(4-7 \mathrm{~Hz})$ band. Results are displayed on the "fiducial cortical surface" (boundary midway through cortical thickness ${ }^{102}$ ). Cortex shown in gray scale, decreased activity in the DOWN compared to the CONTROL condition according to a specific frequency band is labeled red; increased activity is labeled blue. A, anterior; P, posterior; S, superior; I, inferior; $\mathrm{LH}$, left hemisphere; $\mathrm{RH}$, right hemisphere; $\mathrm{BH}$, both hemisphere; $\mathrm{LV}$, left view; $\mathrm{RV}$, right view; $\mathrm{BV}$, bottom view.
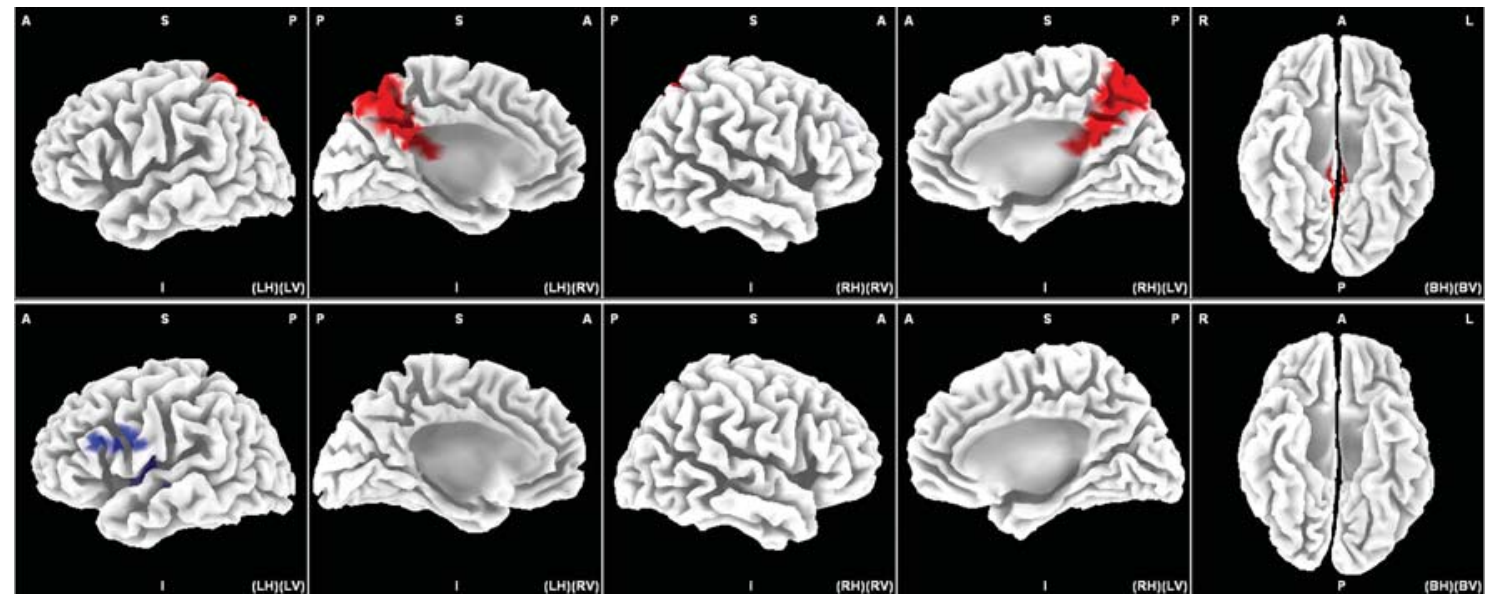

FIG. 7. Low-resolution brain electromagnetic tomography (LORETA) functional images of regional electric activity differences for the comparison "DOWN minus CONTROL" in adolescents. (First row) Alpha (8-12 Hz) band. (Second row) Beta2 (19-21 Hz) band. Legends and color coding as in Figure 6. 
each session would have a different impact on the perceptual and emotional system.

As hypothesized, we found that, compared to the control condition, experiencing a virtual roller coaster ride evoked in both groups, children and adolescents, (1) strong spatial presence experiences, (2) increased skin conductance responses, and (3) with respect to the surface EEG (for which the Alpha band was analyzed) strong event-related power decreases over parietal leads (ERD), indicating cortical activation in this region.

Moreover, children and adolescents showed a different activation pattern at frontal electrode sides. Whereas children revealed event-related power increases (ERS) during the DOWN and END conditions (indicating decreased activation), adolescents consistently showed event-related power decreases (ERD) in the UP, DOWN, and END condition (indicating increased cortical activation over frontal electrodes). Interestingly, this differential cortical activation pattern was also associated with stronger spatial presence experiences in children compared to adolescents. Using LORETA to calculate the intracerebral electrical sources for the surface EEG, we found that the above mentioned EEG activity pattern on the scalp was generated by a distinct network of brain areas known to be involved in visual spatial processing (inferior and superior parietal gyrus, precuneus), the control of executive functions (inferior, middle and superior frontal gyrus), the mapping and regulating of body sensations (insula, postcentral gyrus), and emotion processing (posterior cingulate, cingulate gyrus). The LORETA results mainly confirmed the ERD findings at frontal and parietal electrode positions, however, with increased spatial resolution. Thus, LORETA also revealed that children showed reduced cortical activity, whereas adolescents showed increased cortical activity at frontal brain areas, suggesting that children and adolescents use different neural assemblies to process the visual cues and the spatial presence experience associated with the roller coaster ride.

\section{Activation in the parietal lobe}

As hypothesized, both groups demonstrated the largest ERD values (indicating cortical activation) at parietal electrode positions in the DOWN condition as well as decreased intracerebral electric activity (in the Alpha or Theta band) during the DOWN compared to the CONTROL condition in the posterior cingulate, superior parietal lobe (bilaterally in adolescents), the inferior parietal lobe (right-side in children), and the precuneus (right sided in children). These areas are known to be involved in various steps of spatial processing as well as mental rotation tasks and constitute a neural correlate for the ego-centric view. ${ }^{13-15,18}$ Thus, the spatial cues of the roller coaster scenarios strongly evoked activations within this part of the dorsal processing stream projecting to frontal areas. Obviously, the strength of activation in these areas is important in determining spatial presence experience.

\section{Activation in the insula}

A potential core region for generating spatial presence experiences in an arousing virtual reality world might be the insula for which neural activation changes during the DOWN compared to the CONTROL condition were found in both groups. The insula receives homeostatic afferents from several modalities, including temperature, pain, proprioception, and the viscera and, thus, is involved in the mapping of body related sensations. ${ }^{29}$ In addition, insular activation was also found in several brain imaging studies during aversive emotional stimulation that also evoke visceral/somatic sensations. ${ }^{56}$ Thus, the insula seems to constitute a primary interoceptive image of homeostatic afferents. Interestingly, in children we observed increased neural electric activity bilaterally situated in the insula and the ventral part of the postcentral gyrus (which is also involved in the mapping of body sensations) in the Alpha band, while the adolescents showed increased neural activity in the insula in the Beta2 band. Whereas activity in the Beta2 band can be taken as activation, 55 the Alpha band is (in most paradigms and brain regions) negatively correlated with neural activity as mentioned above. ${ }^{40}$ However, the insula seems to be an exception (i.e., Alpha power in the insula is positively correlated with neural activity). ${ }^{57,58}$ Thus, we conclude that increased insula activity for the Alpha band indicates increased insular activation during the realistic roller coaster ride in children. The reason why adolescents and children differ with respect to the different activation of the Alpha and Beta2 bands is difficult to explain. Perhaps there is a general age-dependent effect on the neural activation pattern in the insula. However, we hypothesize that insula and postcentral gyrus activation reflect increased somatic and visceral reactions during the roller coaster ride compared to the control condition. The importance of somatic and visceral sensations for the experience of spatial presence (in an arousing virtual reality) is further confirmed by the observed positive correlations between skin conductance responses in the UP and 
DOWN conditions and the psychometrical measures of spatial presence. Such positive correlations were also found in psychophysiological studies of spatial presence. 9,11

\section{Activation in the posterior cingulate cortex}

In addition, another brain structure, the cingulate gyrus, is strongly activated in children and adolescents in the DOWN condition (indexed by reduced neural electric activity in the Alpha band). This brain region is involved in various functions, including emotional stimulus processing, 30,52,59 episodic memory function, ${ }^{60}$ and spatial attention ${ }^{61}$ and is strongly connected with frontal and temporal cortical areas. ${ }^{62,63}$ Thus, we hypothesize that the processing centers in the parietal cortex that were strongly activated in this study stimulated the emotional centers via various routes, including the posterior cingulate. These emotional centers (limbic system) might have generated various emotional reactions (including fear, joy, or interest), leading to an increased spatial presence experience in the roller coaster condition.

\section{Activation in the temporal pole}

Children (but not adolescents) showed an increased intracerebral electric activity in the left middle temporal gyrus extending into the temporal pole for the Theta band. This region plays a key role in various functions, including autobiographical and episodic memory, ${ }^{64}$ face recognition, 65 and linguistic as well as emotional language processing. ${ }^{66-68}$ In addition, Theta oscillations in the temporal lobe have been implicated in spatial navigation in animals and in particular have been linked to the encoding and retrieval of spatial information in the hippocampus in rodents. 69,70 Human navigation studies have recently confirmed these findings in showing increased Theta activity over temporal leads.16,71 Related to the present study, these findings might indicate that children encode spatial information during the roller coaster rides. However, we cannot give a plausible explanation why adolescents do not show this activation pattern in the temporal cortex during the roller coaster ride.

\section{Functional dissociation in the frontal cortex between children and adolescents}

Most interesting, as hypothesized children and adolescents showed a clear dissociation with respect to the activation of frontal brain areas, revealed both by the surface EEG analysis (ERD/ERS) as well as by the intracortical electrical analysis (LORETA). Regarding the ERD results, both groups showed in the UP condition (anticipation phase) increased cortical activation at frontal electrodes sides along with the highest skin conductance reactions, indicating highest arousal in the anticipation phase of the high spatial presence condition. In contrast, children demonstrated both in the DOWN and END condition significantly reduced cortical activation at frontal electrode sides, while adolescents showed a similar frontal activation pattern in the UP, DOWN, and END conditions, demonstrating no activation decrease at frontal electrode positions. LORETA confirmed this different frontal activation pattern in children and adolescents in the DOWN compared to the CONTROL condition. Adolescents revealed increased electric activity in the left inferior frontal gyrus and the left precentral gyrus in the Beta2 band. Children on the other hand showed increased electric activity in the right inferior and middle frontal gyrus in the Alpha band as well as increased electric activity in the Theta band bilaterally in the superior frontal gyrus. Based on current knowledge about EEG measurements, increased electric activity in the frontal lobe in the Alpha and Theta bands indicate neural deactivation, while increased electric activity in the Beta2 band indicates increased neural activation. ${ }^{40,55}$ Accordingly, adolescents showed increased neural activation while children on the other hand showed decreased activation in the described frontal structures. Interestingly, these frontal structures are known to be involved in the regulation of various executive functions.

For example, the inferior frontal gyrus is involved in specific functions-including modulating social behavior, ${ }^{72}$ controlling complex sequential actions, ${ }^{73,74}$ simulating or anticipating of actions, ${ }^{75}$ response inhibition, ${ }^{76-80}$ attentional processes, ${ }^{81-84}$ set shifting (Winconsin Card sorting test $\left.{ }^{76,85}\right)$, and detection of incongruities. ${ }^{86}$ However, and more important for the present study, are theoretical assumptions pinpointing the right frontal cortex as a major part of a frontoparietal attention network, including the inferior and middle frontal gyrus as well as the temporo-parietal junction. This network has been shown to operate as a target detection and alarm system directing attention to novel salient events in situations when the system is strongly engaged in processing other stimuli, in this case the virtual reality environment. ${ }^{84}$ Damage to these structures abolishes the P300, which is known to be triggered by the detection of infrequent or novel stimuli embedded in a temporal stream of standard stimuli. 87,88 In addition, it has 
been shown that lesions of the right frontal cortex specifically impair vigilance. ${ }^{89,90}$ Finally, the dorsal parts of the frontal gyrus (superior frontal gyrus) are known to be involved in associating sensory stimuli with motor responses. ${ }^{91-93}$ The prominent role of these areas in processing sensorimotor associations has also been shown by human lesion studies. Patients with lesions in these areas show a kind of "stimulus-driven" behavior characterized by grasping and manually exploring nearly each external stimulus they encounter. ${ }^{94}$

Whether the aforementioned psychological processes are indeed active during the roller coaster ride is difficult to decide. However, we believe that the results of the aforementioned neuroimaging and lesion studies support the idea that adolescents more strongly controlled and monitored the ongoing activities inside and outside the focus of attention, and thus, these mental operations diminished their spatial presence experience. In contrast, decreased activation of these frontal structures in the context of strong activations in parietal areas in younger children might indicate that children were strongly driven by the salient stimuli of the VR environment and neither controlled (or at least to a significant lesser degree) the spatial presence experience nor monitored the surrounding environment. As a consequence, their spatial presence experience was markedly enhanced.

We hypothesize that this reduced frontal activation in children (and consequently reduced executive function) might depend on the non-matured frontal cortex. As mentioned in the introduction, the frontal lobe (especially the white matter) matures until the age of young adulthood.33,34 Thus, the younger children of this study group were examined in a phase during which the frontal cortex is not fully developed and, consequently, the executive functions controlled by this lobe are also not fully functional. Further evidence for this functional "impairment" of the frontal lobe in children comes from behavior and neuroimaging developmental studies. For example, the ability to inhibit prepotent responses markedly increases from childhood into early adulthood, and reaction time improvements for both response execution and response inhibition are observed between the ages of 6 and 20.95,96 Interestingly, neuroimaging studies have shown, in response inhibition tasks (Go/ NoGo), a positive correlation between activation and age in the left inferior frontal gyrus, which was strongly activated in adolescents but not in children in the present roller coaster study. In contrast, children showed in these studies a more extensive activation in discrete regions of the prefrontal cor- tex, indicating an increased demand and inefficient recruitment of brain regions subserving executive functions. ${ }^{97,98}$

Taken together, younger children are obviously more prone to experience spatial presence during a virtual roller coaster drive, due to a not fully developed prefrontal lobe, which leads to reduced control and monitoring of the experimental situation. This anatomical "impairment" might also be the reason for the well-known facts that young children experience strong emotions when watching comic movies, reading books, and playing computer games. Extremely positive or negative emotions can easily be evoked in young children using stimuli that would never evoke such strong emotions in adolescents or adults. ${ }^{99-101}$

\section{Limitations}

Not surprisingly, despite of the promising results, many questions concerning the neurophysiological underpinnings of spatial presence are still unanswered and suggest future studies to further elucidate the functional significance of these findings, especially the functional significance of the dissociation between frontal and parietal cortical structures which seems to play a pivotal role in this study in modulating spatial presence experience. Will the content of any particular environment evoke similar frontal and parietal brain activation? Or can we only observe this frontal activation pattern in passive viewing spatial presence conditions and not in VR worlds where the subjects can interact with the surrounding environment? In other words, if the content of the virtual environment is an everyday situation and not an arousing roller coaster ride, what brain activation pattern can be found? There is no doubt that the brain activation pattern will change significantly if one can freely move around (leading to activation in the motor and premotor cortex) or has to solve cognitive problems (leading at least to activation in the short and long-term memory system and additional parts of frontal cortex) in a VR world. Nevertheless, it might still be the case that subjects who show a decreased activity in some mid-dorsal brain regions and especially some areas of the inferior frontal gyrus will report an increased spatial presence experience.

\section{CONCLUSION}

Summing up, the salient spatial cues delivered by the roller coaster scenarios evoked strong and 
overwhelming activations within the parietal processing centres which in turn stimulated the insula as core region for generation body sensations and the posterior cingulate which is strongly involved in emotion processing. Beside this activation route, there also exists a frontal activation route obviously exerting control over the first stimulus driven route. These frontal areas are involved in controlling the overwhelming and salient spatial and body cues evoked by the roller coaster scenario and are monitoring the ongoing activities outside the focus of processing. Therefore, the results of this study imply that strong spatial presence experiences in an arousing and noninteractive VR world are associated with markedly increased activity in parietal/occipital areas of the brain together with decreased activity in frontal structures involved in the executive system of the brain. In addition, autonomic somatic reactions (e.g., SCR) are increased along with activation in brain structures known to be involved in the somatic and visceral representation of the body state and emotion processing.

\section{ACKNOWLEDGMENTS}

This work was supported by an EU grant supporting the PRESENCE initiative (IST-2001-37661) and the MEC (Measurement, Effect, Conditions) spatial presence project.

\section{REFERENCES}

1. Schubert, T., Friedmann, F., \& Regenbrecht, H. (2001). The experience of presence: factor analytic insights. Presence: Teleoperators and Virtual Environments 10:266-281.

2. Huang, M.P., \& Alessi, N.E. (1999). Presence as an emotional experience. Stud Health Technol Inform 62:148-153.

3. Robillard, G., Bouchard, S., Fournier, T., et al. (2003). Anxiety and presence during VR immersion: a comparative study of the reactions of phobic and non-phobic participants in therapeutic virtual environments derived from computer games. CyberPsychology \& Behavior 6:467-476.

4. Larsson, P., Vastfjall, D., \& Kleiner, M. (2001). The actor-observer effect in virtual reality presentations. CyberPsychology \& Behavior 4:239-246.

5. Flanagan, P., McAnally, K.I., Martin, R.L., et al. (1998). Aurally and visually guided visual search in a virtual environment. Human Factors 40:461-468.

6. Slater, M., Steed, A., McCarthy, J., et al. (1998). The influence of body movement on subjective presence in virtual environments. Human Factors 40:469-477.
7. Mania, K., \& Chalmers, A. (2001). The effects of levels of immersion on memory and presence in virtual environments: a reality centered approach. CyberPsychology \& Behavior 4:247-264.

8. Vastfjall, D. (2003). The subjective sense of presence, emotion recognition, and experienced emotions in auditory virtual environments. CyberPsychology $\mathcal{E}$ Behavior 6:181-188.

9. Meehan, M., Insko, B., Whitton, M., et al. (2002). Physiological measures of presence in stressful virtual environments. ACM Transactions on Graphics 21:645-652.

10. Meehan, M. (2001). Physiological reaction as an objective measure of presence. Chapel Hill: University of North Carolina.

11. Wiederhold, B.K., Jang, D.P., Kim, S.I., et al. (2002). Physiological monitoring as an objective tool in virtual reality therapy. CyberPsychology E Behavior 5:77-82.

12. Laarni, J., Ravaja, N., \& Saari, T. (2004). Measuring mental workload, presence and situation awareness in synthetic environements by eye tracking and psychophysiological methods. In H.M. Khalid, M.G. Helander \& A.W. Yeo (Eds.) Work with Computing Systems. Proceedings of the 7th International Conference on WWCS, p. 475-480. Kuala Lumpur: Damai Sciences.

13. Jordan, K., Schadow, J., Wuestenberg, T., et al. (2004). Different cortical activations for subjects using allocentric or egocentric strategies in a virtual navigation task. Neuroreport 15:135-140.

14. Jordan, K., Heinze, H.J., Lutz, K., et al. (2001). Cortical activations during the mental rotation of different visual objects. Neuroimage. 13:143-152.

15. Jordan, K., Wustenberg, T., Heinze, H.J., et al. (2002). Women and men exhibit different cortical activation patterns during mental rotation tasks. Neuropsychologia 40:2397-2408.

16. Bischof, W.F., \& Boulanger, P. (2003). Spatial navigation in virtual reality environments: an EEG analysis. CyberPsychology \& Behavior 6:487-495.

17. Chouinard, S., Briere, M.E., Rainville, C., et al. (2003). Correlation between evening and morning waking EEG and spatial orientation. Brain Cognition 53: 162-165.

18. Gron, G., Wunderlich, A.P., Spitzer, M., et al. (2000). Brain activation during human navigation: genderdifferent neural networks as substrate of performance. Nat Neurosci 3:404-408.

19. Walter, H., Vetter, S.C., Grothe, J., et al. (2001). The neural correlates of driving. Neuroreport 12:17631767.

20. Uchiyama, Y., Ebe, K., Kozato, A., et al. (2003). The neural substrates of driving at a safe distance: a functional MRI study. Neuroscience Letter 352:199-202.

21. Calhoun, V.D., Pekar, J.J., McGinty, V.B., et al. (2002). Different activation dynamics in multiple neural systems during simulated driving. Human Brain Mapping 16:158-167.

22. Calhoun, V.D., Pekar, J.J., \& Pearlson, G.D. (2004). Alcohol intoxication effects on simulated driving: ex- 
ploring alcohol-dose effects on brain activation using functional MRI. Neuropsychopharmacology 29:20972107.

23. Hoffman, H.G., Richards, T.L., Magula, J., et al. (2003). A magnet-friendly virtual reality fiberoptic image delivery system. CyberPsychology $\mathcal{E}$ Behavior 6:645-648.

24. Mraz, R., Hong, J., Quintin, G., et al. (2003). A platform for combining virtual reality experiments with functional magnetic resonance imaging. CyberPsychology \& Behavior 6:359-368.

25. Schaik, P., Turnbull, T., Wersch, A., et al. (2004). Presence within a mixed reality environment. CyberPsychology \& Behavior 7:540-552.

26. Freeman, J., Avons, S.E., Pearson, D.E., et al. (1999). Effects of sensory information and prior experience on direct subjective ratings of presence. Presence: Teleoperators And Virtual Environments 8:1-13.

27. Ijsselsteijn, W.A., de Ridder, H., Hamberg, R., et al. (1998). Perceived depth and the feeling of presence in 3DTV. Displays 18:207-214.

28. Ijsselsteijn, W.A., de Ridder, H., Freeman, J., et al. (2001). Effects of stereoscopic presentation, image motion, and screen size on subjective and objective corroborative measures of presence. Presence 10: 298-311.

29. Craig, A.D. (2002). How do you feel? Interoception: the sense of the physiological condition of the body. Nat Rev Neurosci 3:655-666.

30. Damasio, A.R., Grabowski, T.J., Bechara, A., et al. (2000). Subcortical and cortical brain activity during the feeling of self-generated emotions. Nat Neurosci 3:1049-1056.

31. Smith, E.E., \& Jonides, J. (1999). Storage and executive processes in the frontal lobes. Science 283:1657-1661.

32. Fuster, J.M. (2002). Frontal lobe and cognitive development. Journal of Neurocytology 31:373-385.

33. Giedd, J.N., Blumenthal, J., Jeffries, N.O., et al. (1999). Brain development during childhood and adolescence: a longitudinal MRI study. Nat Neurosci 2:861-863.

34. Giedd, J.N. (2004). Structural magnetic resonance imaging of the adolescent brain. Ann NY Acad Sci 1021:77-85.

35. Phillips, M.L., Drevets, W.C., Rauch, S.L., et al. (2003). Neurobiology of emotion perception. I. The neural basis of normal emotion perception. Biol Psychiatry 54:504-514.

36. Annett, M. (1970). A classification of hand preference by association analysis. British Journal of Psychology 61:303-321.

37. Lehmann, D. (1990). Past, present and future of topographic mapping. Brain Topography 3:191-202.

38. Lehmann, D. (1988). The view of an EEG-EP mapper. Brain Topography 1:77-78.

39. Laufs, H., Krakow, K., Sterzer, P., et al. (2003). Electroencephalographic signatures of attentional and cognitive default modes in spontaneous brain activity fluctuations at rest. Proc Natl Acad Sci USA 100: 11053-11058.
40. Laufs, H., Kleinschmidt, A., Beyerle, A., et al. (2003). EEG-correlated fMRI of human alpha activity. Neuroimage 19:1463-1476.

41. Gamma, A., Lehmann, D., Frei, E., et al. (2004). Comparison of simultaneously recorded $\left[\mathrm{H}_{2}\left({ }^{15}\right) \mathrm{O}\right]-\mathrm{PET}$ and LORETA during cognitive and pharmacological activation. Human Brain Mapping 22:83-96.

42. Pfurtscheller, G. (1989). Spatiotemporal analysis of alpha frequency components with the ERD technique. Brain Topography 2:3-8.

43. Vorderer, P., Wirth, W., Saari, T., et al. (2004). MEC Spatial Presence Questionnaire (MEC SPQ): short documentation and instructions for application. Report to the European Community, Project Presence: MEC (IST2001-37661). Available Online: http://www.ijk.hmthannover.de/presence.

44. O'Brien, R.G., \& Kaiser, M.K. (1985). MANOVA method for analyzing repeated measures designs: an extensive primer. Psychological Bulletin 97:316-333.

45. Cohen, J. (1969). Statistical power analysis for the behavioral sciences. New York: Academic Press.

46. Holm, S. (1979). A simple sequentially rejective multiple test procedure. Scandinavian Journal of Statistics 6:65-70.

47. Pascual-Marqui, R.D., Lehmann, D., Koenig, T., et al. (1999). Low-resolution brain electromagnetic tomography (LORETA) functional imaging in acute, neuroleptic-naive, first-episode, productive schizophrenia. Psychiatry Research 90:169-179.

48. Pascual-Marqui, R.D., Michel, C.M., \& Lehmann, D. (1994). Low-resolution electromagnetic tomography: a new method for localizing electrical activity in the brain. Int J Psychophysiol 18:49-65.

49. Haalman, I., \& Vaadia, E. (1997). Dynamics of neuronal interactions: relation to behavior firing rates, and distance between neurons. Human Brain Mapping 5:249-253.

50. Talairach, J., \& Tournoux, P. (1988). Co-planar stereotaxic atlas of the human brain. Stuttgart: Thieme.

51. Towle, V.L., Bolanos, J., Suarez, D., et al. (1993). The spatial location of EEG electrodes: locating the bestfitting sphere relative to cortical anatomy. Electroencephalogr Clin Neurophysiol 86:1-6.

52. Esslen, M., Pascual-Marqui, R.D., Hell, D., et al. (2004). Brain areas and time course of emotional processing. Neuroimage. 21:1189-1203.

53. Pascual-Marqui, R.D., Esslen, M., Kochi, K., et al. (2002). Functional imaging with low-resolution brain electromagnetic tomography (LORETA): a review. Methods Find Exp Clin Pharmacol 24:91-95.

54. Kubicki, S., Herrmann, W.M., Fichte, K., et al. (1979). Reflections on the topics: EEG frequency bands and regulation of vigilance. Pharmakopsychiatr Neuropsychopharmakol 12:237-245.

55. Oakes, T.R., Pizzagalli, D.A., Hendrick, A.M., et al. (2004). Functional coupling of simultaneous electrical and metabolic activity in the human brain. Human Brain Mapping 21:257-270.

56. Phan, K.L., Wager, T., Taylor, S.F., et al. (2002). Functional neuroanatomy of emotion: a meta-analysis of 
emotion activation studies in PET and fMRI. Neuroimage 16:331-348.

57. Goldman, R.I., Stern, J.M., Engel, J., Jr., et al. (2002). Simultaneous EEG and fMRI of the alpha rhythm. Neuroreport 13:2487-2492.

58. Sadato, N., Nakamura, S., Oohashi, T., et al. (1998). Neural networks for generation and suppression of alpha rhythm: a PET study. Neuroreport 9:893-897.

59. Maddock, R.J., \& Buonocore, M.H. (1997). Activation of left posterior cingulate gyrus by the auditory presentation of threat-related words: an fMRI study. Psychiatry Research 75:1-14.

60. Maddock, R.J., Garrett, A.S., \& Buonocore, M.H. (2003). Posterior cingulate cortex activation by emotional words: fMRI evidence from a valence decision task. Human Brain Mapping 18:30-41.

61. Mesulam, M.M., Nobre, A.C., Kim, Y.H., et al. (2001). Heterogeneity of cingulate contributions to spatial attention. Neuroimage 13:1065-1072.

62. Morris, R., Pandya, D.N., \& Petrides, M. (1999). Fiber system linking the mid-dorsolateral frontal cortex with the retrosplenial/presubicular region in the rhesus monkey. J Comp Neurol 407:183-192.

63. Allison, T., Puce, A., \& McCarthy, G. (2000). Social perception from visual cues: role of the STS region. Trends in Cognitive Science 4:267-278.

64. Maguire, E.A., \& Mummery, C.J. (1999). Differential modulation of a common memory retrieval network revealed by positron emission tomography. Hippocampus 9:54-61.

65. Sergent, J., Ohta, S., \& MacDonald, B. (1992). Functional neuroanatomy of face and object processing. A positron emission tomography study. Brain 115: 15-36.

66. Braak, H., Braak, E., Yilmazer, D., et al. (1996). Functional anatomy of human hippocampal formation and related structures. J Child Neurol 11:265-275.

67. Tzourio, N., Crivello, F., Mellet, E., et al. (1998). Functional anatomy of dominance for speech comprehension in left handers vs right handers. Neuroimage 8:1-16.

68. Dupont, S. (2002). Investigating temporal pole function by functional imaging. Epileptic Disorders 4:S17-S22.

69. Skaggs, W.E., McNaughton, B.L., Wilson, M.A., et al. (1996). Theta phase precession in hippocampal neuronal populations and the compression of temporal sequences. Hippocampus 6:149-172.

70. O'Keefe, J., \& Recce, M.L. (1993). Phase relationship between hippocampal place units and the EEG theta rhythm. Hippocampus 3:317-330.

71. Kahana, M.J., Sekuler, R., Caplan, J.B., et al. (1999). Human theta oscillations exhibit task dependence during virtual maze navigation. Nature 399:781-784.

72. Decety, J., \& Chaminade, T. (2003). Neural correlates of feeling sympathy. Neuropsychologia 41:127-138.

73. Corballis, M.C. (1991). The lopsided ape. New York: Oxford University Press.

74. Corballis, M.C. (1992). On the evolution of language and generativity. Cognition 44:197-226.
75. Adam, J.J., Backes, W., Rijcken, J., et al. (2003). Rapid visuomotor preparation in the human brain: a functional MRI study. Brain Res $\operatorname{Cog} n$ Brain Res 16:1-10.

76. Konishi, S., Nakajima, K., Uchida, I., et al. (1999). Common inhibitory mechanism in human inferior prefrontal cortex revealed by event-related functional MRI. Brain 122:981-991.

77. Rubia, K., Smith, A.B., Brammer, M.J., et al. (2003). Right inferior prefrontal cortex mediates response inhibition while mesial prefrontal cortex is responsible for error detection. Neuroimage 20:351-358.

78. Taylor, S.F., Phan, K.L., Decker, L.R., et al. (2003). Subjective rating of emotionally salient stimuli modulates neural activity. Neuroimage 18:650-659.

79. Taylor, S.F., Kornblum, S., Lauber, E.J., et al. (1997). Isolation of specific interference processing in the Stroop task: PET activation studies. Neuroimage 6: 81-92.

80. Menon, V., Adleman, N.E., White, C.D., et al. (2001). Error-related brain activation during a Go/NoGo response inhibition task. Human Brain Mapping 12: 131-143.

81. Jancke, L., \& Shah, N.J. (2002). Does dichotic listening probe temporal lobe functions? Neurology 58 : 736-743.

82. Noesselt, T., Shah, N.J., \& Jancke, L. (2003). Topdown and bottom-up modulation of language related areas-an fMRI Study. BMC Neuroscience 4:13.

83. Heyder, K., Suchan, B., \& Daum, I. (2004). Corticosubcortical contributions to executive control. Acta Psychol (Amst) 115:271-289.

84. Corbetta, M., \& Shulman, G.L. (2002). Control of goal-directed and stimulus-driven attention in the brain. Nat Rev Neurosci 3:201-215.

85. Nakahara, K., Hayashi, T., Konishi, S., et al. (2002). Functional MRI of macaque monkeys performing a cognitive set-shifting task. Science 295:1532-1536.

86. Moran, J.M., Wig, G.S., Adams, R.B., Jr., et al. (2004). Neural correlates of humor detection and appreciation. Neuroimage 21:1055-1060.

87. Daffner, K.R., Mesulam, M.M., Scinto, L.F., et al. (2000). The central role of the prefrontal cortex in directing attention to novel events. Brain 123:927-939.

88. Knight, R.T., \& Scabini, D. (1998). Anatomic bases of event-related potentials and their relationship to novelty detection in humans. J Clin Neurophysiol 15:3-13.

89. Wilkins, A.J., Shallice, T., \& McCarthy, R. (1987). Frontal lesions and sustained attention. Neuropsychologia 25:359-365.

90. Daffner, K.R., Mesulam, M.M., Holcomb, P.J., et al. (2000). Disruption of attention to novel events after frontal lobe injury in humans. J Neurol Neurosurg Psychiatry 68:18-24.

91. Jancke, L., Loose, R., Lutz, K., et al. (2000). Cortical activations during paced finger-tapping applying visual and auditory pacing stimuli. Brain Res Cogn Brain Res 10:51-66.

92. Lutz, K., Specht, K., Shah, N.J., et al. (2000). Tapping movements according to regular and irregular visual 
timing signals investigated with fMRI. Neuroreport 11:1301-1306.

93. Toni, I., Schluter, N.D., Josephs, O., et al. (1999). Signal-, set- and movement-related activity in the human brain: an event-related fMRI study. Cerebral Cortex 9:35-49.

94. Heilman, K.M., Valenstein, E. (2003). Clinical neuropsychology. New York: Oxford University Press.

95. Band, G.P., van der Molen, M.W., Overtoom, C.C., et al. (2000). The ability to activate and inhibit speeded responses: separate developmental trends. J Exp Child Psychol 75:263-290.

96. Williams, B.R., Ponesse, J.S., Schachar, R.J., et al. (1999). Development of inhibitory control across the life span. Dev Psychol 35:205-213.

97. Rubia, K., Overmeyer, S., Taylor, E., et al. (2000). Functional frontalisation with age: mapping neurodevelopmental trajectories with fMRI. Neurosci Biobehav Rev 24:13-19.

98. Tamm, L., Menon, V., \& Reiss, A.L. (2002). Maturation of brain function associated with response inhibition. J Am Acad Child Adolesc Psychiatry 41: 1231-1238.

99. Luna, B., Thulborn, K.R., Munoz, D.P., et al. (2001). Maturation of widely distributed brain function subserves cognitive development. Neuroimage 13: 786-793.
100. Ridderinkhof, K.R., \& Vandermolen, M.W. (1995). A psychophysiological analysis of developmental differences in the ability to resist interference. Child Development 66:1040-1056.

101. Bjorklund, D.F., \& Harnishfeger, K.K. (1990). The resources construct in cognitive-development-diverse sources of evidence and a theory of inefficient inhibition. Developmental Review 10:48-71.

102. Dickson, J., Drury, H., \& Van Essen, D.C. (2001). "The surface management system" (SuMS) database: a surface-based database to aid cortical surface reconstruction, visualization and analysis. Philos Trans R Soc Lond B Biol Sci 356:1277-1292.

\author{
Address reprint requests to: \\ Thomas Baumgartner, Ph.D. \\ Institute for Empirical Research \\ in Economics and Neuroeconomics \\ University of Zurich \\ Blümlisalpstrasse 10 \\ CH-8006 Zürich \\ Switzerland
}

E-mail: t.baumgartner@iew.unizh.ch 